\title{
P50 Gating is Not Affected by Selective Attention
}

\author{
Kuan H. Kho ${ }^{1,2}$, Robbert J. Verkes ${ }^{2}$, Paul Eling ${ }^{1}$, Machiel J. Zwarts ${ }^{3}$, \\ Bart Ellenbroek ${ }^{4}$, and Gilles van Luijtelaar ${ }^{1}$
}

\begin{abstract}
Accepted for publication: December 2, 2002
${ }^{1} \mathrm{NICl}$, Dept. of Biological Psychology, University of Nijmegen The Netherlands, ${ }^{2}$ Dept. of Psychiatry, University Medical Centre Nijmegen, The Netherlands, ${ }^{3}$ Dept. of Clinical Neurophysiology, University Medical Centre Nijmegen, The Netherlands, ${ }^{4}$ Dept. of Psychoneuropharmacology, University Medical Centre Nijmegen, The

Netherlands

Keywords: AEP, auditory evoked potentials, sensory gating, P50, P1, P50 gating, selective attention, N100

Abstract The influence of selective attention on P50 gating - the decline of the amplitude of the P50 component of the auditory evoked potential (AEP) to the second of a pair of clicks - was examined. Three conditions were presented in counterbalanced order to 29 healthy volunteers: a baseline condition, in which the double click was presented and no specific task was required, an attention condition in which attention to the clicks was required, and a distraction condition in which paired clicks were presented during rehearsal time of a reversed digit span task. P50 gating, as measured with ratio and difference scores, did not differ across the three conditions. However, the amplitude of the N100 showed an increase in the attention condition compared to the two nonattention conditions, supporting the validity of our task manipulations. The data on P50 demonstrate that P50 gating is not affected by attentional manipulations.
\end{abstract}

\section{Introduction}

The suppression of the amplitude of the $\mathrm{P} 50(\mathrm{~Pb}$ or $\mathrm{P} 1)$ of the auditory evoked potential (AEP) in click pairs has been the focus of attention in both animal (van Luijtelaar et al., 1998; Ellenbroek et al., 2000; De Bruin et al., 2001) and clinical studies (Adler et al., 1982; Freedman et al., 1987; Judd et al., 1992). In a double-click paradigm two identical clicks are presented with a short $(500 \mathrm{~ms})$ interval. There is a reduction in amplitude of the P50 of the second of the paired clicks in normal subjects, reflecting a slow inhibitory process. However, in schizophrenic patients the response to the second click is comparable with the response to the first or only minimally reduced. This phenomenon has been interpreted as a reflection of impaired sensory gating (Adler et al. 1982; Nagamoto, Adler, Waldo, \& Freedman, 1989; Freedman et al., 1987). It can be speculated that the absence of the decline in amplitude to the second click in a episode of 500 to $1000 \mathrm{~ms}$ after stimulus presentation reflects neuronal impairment of auditory sensory gating, which results in flooding the brain with redundant information, and that this disturbance might be involved in the cognitive deficit that occurs in schizophrenia.
The P50 component is generally considered exogenous, i. e., insensitive to cognitive operations. AEP components that occur $100 \mathrm{~ms}$ or more after stimulus presentation are endogenous and under the influence of cognitive processes (Coles \& Rugg, 1995). Although the P50 potential is thought to be insensitive to attentional variables, (Freedman et al., 1987; Waldo \& Freedman, 1986), the findings regarding attentional influences on P50 gating are contradictory. P50 gating is not affected by manipulations of attention according to some (Jerger, Biggins, \& Fein, 1992; White \& Yee, 1997) and is therefore considered by these authors as preattentive. Others have found that P50 gating is reduced when subjects are instructed to pay attention to the stimuli (Guterman, Josiassen, \& Bashore, 1992; Guterman \& Josiassen, 1994). It is important to establish the effects of attention on P50 gating in order to interpret suppression of P50 gating in subjects with various psychopathologies. Therefore, the general question is whether gating is a preattentive, "hardwired," neuronal slow inhibitory process (Jerger et al., 1992; White \& Yee, 1997), or a manifestation of a process under the influence of attention (Guterman et al., 1992; Guterman \& Josiassen, 1994).

The reasons for the different results reported in the 
literature are not clear. The study conducted by Jerger et al. (1992) showed that P50 gating was comparable in the two tasks that required attention whereas the amplitude of the N100 of the second click was larger. The N100 is considered a direct index for selective attention; its amplitude is positively related to the amount of attention invested (Hilyard et al., 1973; Coull, 1998). The presentation of the N100 data assists in interpreting whether attention was successfully manipulated or not. White and Yee (1997) found no effects of attention on P50 gating, compared to a baseline condition. However, in a second experiment in which they compared a stress with a baseline condition, a change in P50 gating was found. In a subsequent experiment they found that competing cognitive activity, as opposed to a resting, baseline state, did not elicit differences in P50 gating (Yee \& White, 2001). However, no N100 amplitudes and changes were reported. Guterman et al. (1992) applied four conditions that differed in the amount of selective attention to the auditory stimuli: Their analyses showed differences in P50 gating as well as in N100 amplitude across the conditions. In this study the conditions in which attention was modulated resembled the "stress" condition used by White and Yee (1998). The aim of the present study was to further elucidate the influence of selective attention on P50 gating in a within-subjects procedure, which included both a baseline (resting) condition, an attention condition, and a distraction condition. Moreover, we included the amplitude of the N100 in the analysis in order to demonstrate that attention was successfully manipulated.

\section{Methods}

\section{Subjects}

Twenty-nine healthy (11 M, $18 \mathrm{~F})$ volunteers participated as subjects; their age was between 18 and 47 years. Individuals were excluded if they had a history of significant medical, neurological, or psychiatric illness, including alcohol and substance abuse, or the presence of such disorders in first-degree relatives. Smokers were excluded as well. A further constraint was that no relevant medical illness had been present, and that no medication had been used in the month before testing, and that no coffee or tea had been consumed in the last 12 hours before testing. The study was approved by the Human Ethics Committee of the UMC Nijmegen. Subjects gave informed consent after full explanation of the study procedures. Electroencephalograms (EEGs) were obtained purely for research purposes.

\section{Experimental Design}

Auditory pairs of clicks were presented through headphones. The stimuli were created and generated by the Neuroscan Stim system. The clicks had a duration of $0.2 \mathrm{~ms}$ and an intensity of $80 \mathrm{~dB}$. The interval between the first and the second pulse was $500 \mathrm{~ms}$. The intertrial interval (interpair interval) varied randomly between 8 and $10.5 \mathrm{~s}$. Eight practice trials were presented to familiarize the subjects with the stimuli and to ensure no startle response was elicited. Subsequently, 84 trials were presented three times (three conditions). In $20 \%$ of all auditory stimulus pairs, a click-tone pair was presented instead of a click pair; these were pseudorandomly distributed across trials.

The participants were exposed to three conditions. In the baseline condition, the subject was instructed to sit back and relax, but nothing was said about the presentation of the auditory stimuli. In the attention condition, the subject was instructed to attend to the tones, and to count them silently. Subjects were asked to report the total number of tones after completion of the first 42 trials and after the second 42 trials. A discrepancy of more than two between the answer and the actual number of tones was considered to indicate a failure to comply with the task instructions. In the distraction condition, the subjects were engaged in a reversed digit-span task when the auditory stimuli were presented. The digits (white digits, background black, 1 s/digit) were visually presented, and the subject was instructed to memorize and to recall the digits in reversed order after an imperative stimulus. The screen turned blank after the last digit in the sequence, the subjects had to rehearse the digits and in the meantime three click-pairs were presented, just as in the other two conditions. Next, the imperative stimulus was presented for $1 \mathrm{~s}$ : a solid green circle in the middle of the screen against a black background. After $5 \mathrm{~s}$, another digit sequence appeared, and the cycle started again. The responses of the subjects were recorded verbatim. Prior to this condition, subjects were tested on the digits backward task to assess their memory span. This span was then used, while on $5 \%$ of the trials one digit less and on 5\% trials one digit more were presented to the subjects, in order to both motivate the subjects and keep them alert.

The order in which the three conditions were presented was counterbalanced. The instructions stressed that subjects should pay attention to the auditory stimuli in the attention condition, whereas in the distraction condition subjects were told that their memory span was being measured, and that the auditory stimuli were distractors they need not attend to. 


\section{Signal Recording}

Electroencephalogram recordings were made with $\mathrm{Ag} / \mathrm{AgCl}$ cup electrodes from $\mathrm{Fz}, \mathrm{Cz}$, and $\mathrm{Pz}$ (International 10-20 system, Jasper, 1958), and according to international guidelines (Picton et al., 2000). Linked mastoids were used as reference. The bandpass was between 0.53 and $500 \mathrm{~Hz}$. Horizontal electro-oculogram (EOG) recordings were made from tin electrodes placed at the outer canthus of each eye. Similarly, vertical EOG was recorded from electrodes placed infra- and supraorbital to the left eye. The bandpass for EOG was between 0.032 and $100 \mathrm{~Hz}$. A ground electrode was attached to the left temporal-parietal scalp. We attached the electrodes using electrode paste (Ten 20 Conductive EEG paste, D.O. Weaver \& Co., Aurora, CO, USA) after cleaning the skin with alcohol (90\%) and scrubbing with Nuprep ECG \& EEG Abrasive Skin Prepping Gel (D.O. Weaver \& Co., Aurora, CO, USA) to keep impedance below $5 \mathrm{k} \Omega$. The sample rate was $2000 \mathrm{~Hz}$.

\section{Data Analysis}

The EEG and EOG data of the three conditions were analyzed using a software package especially designed for EEG and AEP data processing (Neuroscan Edit). First, all of the sampled EEG epochs were baseline (100 ms baseline) corrected. Subsequently, all epochs containing vertical EOG movements or muscle activity (amplitudes $>100 \mu \mathrm{V}$ ) were removed. The epochs were filtered offline at $70 \mathrm{~Hz}$.

Peaks scoring was done manually. The P50-N100 complex was visually identified, where the P50 was defined as the positive peak preceding the large N100 component. The P50 was measured relative to the preceding through, the $\mathrm{Nb}$. This is a common procedure used in P50 gating research which was first explicitly described by Nagamoto et al. (1989). An auxiliary criterion was used for scoring the peak after the second stimulus: It should occur $500 \pm 10 \mathrm{~ms}$ after the 1st P50 (Nagamoto et al., 1989).

P50 gating measures were calculated by (1) subtracting the P50 amplitude S2 from S1 (e. g., Smith, Boutros, \& Schwarzkopf, 1994); (2) the ratio between S2 and S1 (S2/S1).

\section{Statistical Analysis}

The dependent variables were evaluated by means of repeated measure MANOVA's for the N100 amplitude, the P50 amplitude, and for the two gating measures. Both
CLICK (2 levels, S1 versus S2) and CONDITION (baseline, attention, distraction) were treated as within-subjects factors, with order of condition (6 levels: $\mathrm{ABC}, \mathrm{ACB}$, $\mathrm{BAC}, \mathrm{BCA}, \mathrm{CAB}, \mathrm{CBA})$ as a between-subject factor.

To assess test-retest reliability of the P50 amplitudes as such, intraclass correlation coefficients (ICC) of the amplitude of the P50's and its gating measures across the three conditions were calculated (Bartko \& Carpenter, 1976; Smith et al., 1994). The ICC is in fact the variance of the measures between individuals, compared to the total variance, i. e., the sum of the variance within and between individuals.

Pearson correlation coefficients between the conditions for each P50 gating measure and P50 amplitudes were calculated as well. However, the correlation coefficients should not be extremely high since some systematic variance was introduced by having six different orders.

\section{Results}

None of the subjects made a substantial number of errors in the distraction condition or miscounted the number of stimuli by more than two in the attention condition. None of the subjects reported afterward, even if asked, that they were unable to comply with the task instructions. Therefore, all data were included in the overall statistical analyses.

The P50 was most often best expressed at Cz, although clear P50 was also seen at Fz or Pz. Figure 1 shows the grand average AEP in the three conditions. The mean and standard deviations of the amplitude of the P50 and N100 at Cz, as well as the variables that indicate gating, are shown for the three conditions in Table 1. Analysis of the amplitude of the $N 100$ revealed a significant effect for CLICK $[F(1,23)=58.47, P<.001]$ and CONDITION $[F(2,22)=8.76, P<.01]$. Interactions betweenCONDITION $\times$ ORDER $[F(10,44)=4.96, P<.001]$ and CLICK $\times$ CONDITION $\times$ ORDER $[F(10,44)=4.17, P<.001]$ were additionally found. Post-hoc analysis of CONDITION (Bonferroni corrected) revealed that the amplitude of the N100 was larger in the attention condition compared to the baseline and the distraction conditions; post-hoc tests on the interactions demonstrated that the amplitude of the N100 was larger if the attention condition was presented as the first condition rather than as the last condition. Gating of the N100 did occur (significant CLICK effect), although there was no interaction between click and condition, suggesting that attention did not affect N100 gating.

The multivariate repeated measures analysis of variance on the amplitude of the P5O showed a significant 
Table 1 Means and standard deviation (SD) of the amplitude (in $\mu \mathrm{V}$ ) of the P50, two measures of P50 gating and the amplitude of the $\mathrm{N} 100$.

\begin{tabular}{|c|c|c|c|c|}
\hline & $\begin{array}{l}\text { Baseline } \\
\text { condition } \\
\text { Mean SD }\end{array}$ & $\begin{array}{l}\text { Attentio } \\
\text { conditio } \\
\text { Mean }\end{array}$ & & $\begin{array}{l}\text { Distraction } \\
\text { condition } \\
\text { Mean SD }\end{array}$ \\
\hline \multicolumn{5}{|l|}{ P50 } \\
\hline S1 & 3.132 .32 & 3.73 & 2.43 & 3.412 .40 \\
\hline S2 & 1.801 .60 & 2.34 & 1.69 & 2.402 .30 \\
\hline $\mathrm{S} 1-\mathrm{S} 2$ & 1.331 .71 & 1.39 & 1.82 & 1.011 .75 \\
\hline $\mathrm{S} 2 / \mathrm{S} 1$ & $\begin{array}{lll}0.71 & 0.71\end{array}$ & 0.78 & 0.58 & 0.860 .76 \\
\hline \multicolumn{5}{|l|}{ N100 } \\
\hline S1 & -16.168 .91 & -18.25 & 10.51 & -14.087 .90 \\
\hline S2 & - 5.872 .81 & -8.03 & 3.65 & - 5.732 .54 \\
\hline
\end{tabular}

Table 2 Pearson correlation and intraclass correlation coefficients of P50 amplitudes and P50 gating measures.

\begin{tabular}{lll}
\hline & $\begin{array}{l}\text { Attention } \\
\text { condition }\end{array}$ & $\begin{array}{l}\text { Distraction } \\
\text { condition }\end{array}$ \\
\hline P50 S1 & $0.83^{* *}$ & $0.72^{* *}$ \\
Baseline condition & $0.68^{* *}$ \\
Attention condition & ICC & 0.74 \\
Distraction condition & & \\
\hline P50 S2 & $0.60^{* *}$ & $0.60^{* *}$ \\
Baseline condition & & 0.30 \\
Attention condition & ICC & 0.45 \\
Distraction condition & & \\
\hline S1-S2 & $0.40^{*}$ & $0.45^{*}$ \\
Baseline condition & & $0.48^{* *}$ \\
Attention condition & ICC & 0.45 \\
Distraction condition & & \\
\hline S2/S1 & & $0.63^{* *}$ \\
Baseline condition & -0.13 & 0.22 \\
Attention condition & & 0.29 \\
Distraction condition & ICC &
\end{tabular}

$P<.05^{* *} P<.01$

effect of CLICK $[F(1,23)=21.95, P<.001]$ and CONDITION $[F(2,22)=6.28, P<.05]$. There were no significant effects for order of condition or for interactions. The significant CLICK effect and the larger amplitude on the first compared to the second click indicates that gating occurred. Moreover, a significant effect of condition on the amplitudes of the P50 was found. Post-hoc analysis showed that the baseline condition differed from the attention condition ( $P=0.024$, Bonferroni corrected), though no significant difference was found between baseline and distraction conditions, nor between attention and distraction conditions.

All subjects were included in the analyses of P50 gat-

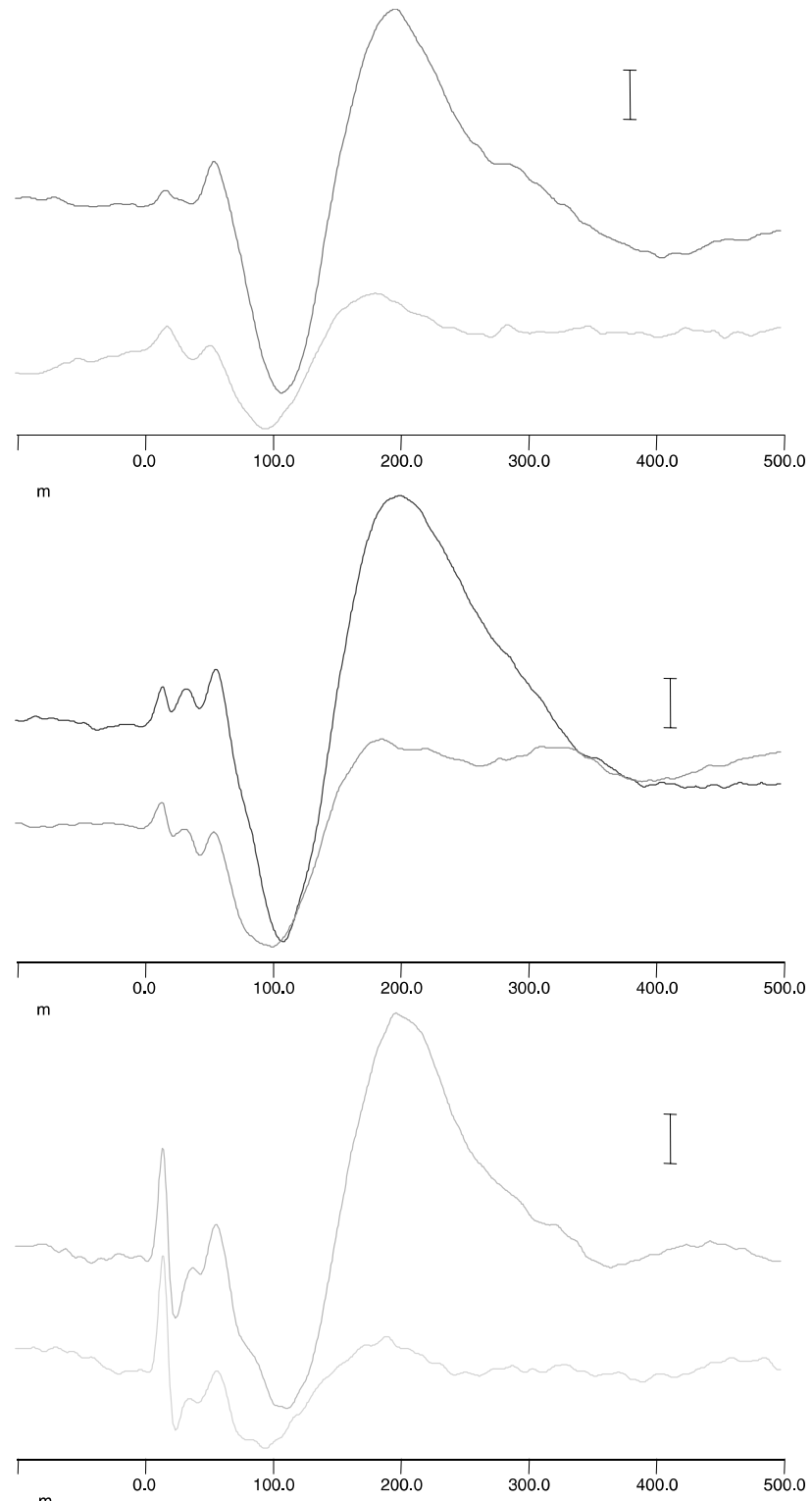

Figure 1 Grand average of the AEP's in the three conditions to the first (large potential) and second (small potential) click. Top: baseline condition, middle: attention condition, bottom: distraction condition. Note the presence of a myogene response (at 17 ms after stimulus onset) in the lower trace.

ing, and no truncation of outliers took place. The two $P 50$ gating measures (S1-S2 and S2/S1) were analyzed, but neither of them showed any main effects or an interaction between CONDITION and ORDER. Using an $\alpha$ of 0.05 , given the variances observed and the sample size of 29 subjects, the statistical tests used had a power of 0.80 to discern between any two of the conditions a difference of minimally 0.90 for the S1-S2 and a difference of 0.37 for the $\mathrm{S} 2 / \mathrm{S} 1$.

The Pearson correlation coefficients and the ICC's of the P50 amplitudes and the gating measurements are presented in Table 2. 


\section{Discussion}

The attention condition showed a higher amplitude of the N100 compared to the baseline and distraction conditions. This result is consistent with data reported in the literature, in which it is generally found that the amplitude N100 is higher under conditions of selective attention (Hillyard et al., 1973; Mangun \& Hillyard, 1995). The amplitude of the N100 can be considered as a marker for attention. Our findings concerning the amplitude of the N100 validate our task manipulations. However, the cLICK effect demonstrating gating of the $\mathrm{N} 100$ and also the interaction between ORDER and CONDITION show that attention and task instruction are not the only factors that influence the amplitude of the N100. Since the amplitude of the N100 tended to be higher in the first condition than in the other two conditions, it seems that novelty and fatigue also modulate the amplitude of the N100. Interestingly, although gating was found to occur for the $\mathrm{N} 100$, the lack of interaction between condition and click suggests that N100 gating is not influenced by attention.

A preliminary and surprising finding was that the amplitudes of the P50 differed in the three conditions. The interpretation of this finding is unclear, however, as there seems to be no difference between the distraction (and perhaps demanding) condition, on the one hand, and the baseline and attention conditions, on the other hand only between the baseline and attention conditions. Perhaps the amplitude of the P50 is under the influence of higher cognitive processes. Attention effects in the middle-latency range (12-50 ms from stimulus onset) appear to occur under some conditions, but it is not clear whether these effects are exogenous or endogenous (Näätänen $\&$ Teder, 1991). Also Hackley's review (1993) on evoked potentials and selective attention shows that in some studies attention could modify potentials as early as $30 \mathrm{~ms}$. The difference between the effects of attention on the amplitude of N100 and P50, (attention > baseline and distraction vs attention > baseline, respectively) might be due to a larger signal to noise ratio for the two components: The amplitude of the P50 is much smaller than that of the N100.

Task manipulation did not influence $P 50$ gating. Gating occurred in all three conditions, and the lack of interaction between CLICK and CONDITION demonstrates that neither selective attention nor distraction had an effect on P50 gating in healthy volunteers. This lack of a condition effect seems quite reliable considering the large number of subjects used; more sensitive tests (paired $t$-tests) did not reveal a difference (even a small one) between any of the three conditions, and the difference in gating between any of the conditions had to be .9 instead of the obtained $.38, .32$ or .6 for S1-S2 and .37 instead of $.15, .8$ and .7 for the S1/S2. The present finding - no effect of attention on P50 gating - is consistent with the view that gating is a preattentive phenomenon. The distraction condition of our study required the participant not only to attend to a different modality (visual), but also to memorize and process the digits in order to recall them in the reversed order. The complexity of this task distracts subjects from the auditory stimuli. It can perhaps also be regarded as a somewhat demanding task, comparable to the "stress" condition used in the White and Yee study (1997). They found a reduction in P50 gating when the stress condition was compared to the baseline condition. Disturbances in P50 gating were also found in Post Traumatic Stress Disorder patients (Neylan et al., 1999). However, in the present study a reduction in gating was not found in the distraction condition, suggesting that the complexity of the task did not induce sufficient amounts of stress in our subjects to reduce gating. This might be due to the fact that the level of difficulty of this task was individually adapted in our study. Moreover, it was found recently that P50 gating is not affected by cognitive activity (Yee \& White, 2001). However, no N100 amplitudes were reported which could have provided information about the amount of selective attention that was directed to the auditory stimuli. The contradictory results of Guterman and Josiassen (1994) (see Introduction) could be partly explained by a confounding effect due to the requirement to respond: Anticipation and preparation of a motor response were required in their study. Moreover, Yee and White (2001) also suggest that heightened facial muscle activity and strong task demands have modulatory effects on P50 suppression. Other studies investigating P50 gating and levels of arousal indicate that gating in rats as well in humans is not always altered across different states of arousal and sleep (van Luijtelaar et al., 1998; Kisley, Olincy, \& Freedman, 2001). The P50 gating process, therefore, seems to be a robust preattentive phenomenon.

The value of the ICC for the P50 amplitude after the first click is consistent with the findings of Arnfred et al. (2001a). It indicates that the amplitude of the P50 is robust and reliable within subjects. The ICC was lower for the S2, suggesting that is was less reliable and more variable within subjects. Also the ICC of gating as expressed by the S2/S1 ratio found in the present study (also described as T/C ratio) was lower than that obtained by Arnfred et al. (2001b), although it exceeded the ratios found by others (e. g., Freedman et al., 1987; Waldo et al., 1986). However, this measure as such is prone to large deviations, not uncommon for ratios. The ICC of gating calculated as the difference between S1 and S2 is higher than the ICC of the ratio score and 
seems therefore to be preferable to a ratio score (see also Smith et al., 1994). Based on the values of both the product-moment and intracorrelation coefficient, we consider that the consistency of the measures over the three conditions is sufficient and comparable to what others have reported.

The double-click paradigm and P50 gating can be considered a valid measure in clinical research (e.g., on schizophrenia) to establish whether preattentive processes are disturbed. However, it is doubtful whether gating in the auditory modality is strongly correlated with gating in other modalities (Adler et al., 1985), and whether there is a relationship between disturbed gating in the somatosensory and the auditory modality in healthy volunteers and schizophrenic patients (Arnfred et al., 2001a). Until now, there was little evidence of crossmodal correlation of normal and disturbed.

It can be concluded that auditory sensory gating seems to be a unique and quite reliable paradigm for measuring automatic and preattentive processes.

\section{References}

Adler, L.E., Pachtman, E., Franks, R.D., Pecevich, M., Waldo, M.C., \& Freedman, R. (1982). Neurophysiological evidence for a defect in neuronal mechanisms involved in sensory gating in schizophrenia. Biological Psychiatry, 17, 639-654.

Adler, L.E., Waldo, M.C., \& Freedman, R., (1985). Neurophysiological studies of sensory gating in schizophrenia: Comparison of auditory and visual responses. Biological Psychiatry 20, 1284-1296.

Arnfred, S.M., Eder, D.N., Hemmingsen, R.P., Glenthøj, B.Y., \& Chen, A.C.N. (2001a). Gating of the vertex somatosensory and auditory evoked potential P50 and the correlation to skin conductance orienting response in healthy men. Psychiatry Research, 101, 221-235.

Arnfred, S.M., Chen, A.C.N., Eder, D.N., Glenthøj, B.Y., \& Hemmingsen, R.P. (2001b). A mixed modality paradigm for recording somatosensory and auditory P50 gating. Psychiatry Research, 105, 79-86.

Bartko, J.J., \& Carpenter, W.T. (1976). On methods and theory of reliability. The Journal of Nervous and Mental Disease, 163, 307-317.

Coles, M.G.H., \& Rugg, M.D. (Eds.). (1995). Electrophysiology of mind. Event-related brain potentials and cognition. Oxford: Oxford University Press.

Coull, J.T. (1998). Neural correlates of attention and arousal: Insights from electrophysiology, functional neuroimaging and psychopharmacology. Progress in Neurobiology, 55, 343-361.

De Bruin, N.M.W.J., Ellenbroek, B.A., Cools, A.R., Coenen, A.M.L., \& van Luijtelaar, E.L.J.M. (1999). Differential effects of ketamine on gating of auditory evoked potentials and prepulse inhibition in rats. Psychopharmacology, 142, 9-17.

Dupont, W.P., \& Plummer W.D. (1990). Power and sample size calculations: Areview and computer program. Controlled Clinical Trials, 11, 116-128.
Ellenbroek, B.A., Van Luijtelaar, G., Frenken, M., \& Cools, A.R. (1999). Sensory gating in rats: Lack of correlation between auditory evoked gating and prepulse inhibition. Schizophrenia Bulletin, 25, 777-788.

Ellenbroek, B.A., Sams-Dodd, F., \& Cools, A.R. (2000). Simulation models for schizophrenia. In B.A. Ellenbroek, \& A.R. Cools (Eds.), Atypical antipsychotics. Basel: Birkhauser Verlag.

Hackley, S.A. (1993). An evaluation of the automaticity of sensory processing using event-related potentials and brain stem reflexes. Psychophysiology, 30, 415-428.

Hillyard, S.A., Hink, R.F., Schwent, V.L., \& Picton, T.W. (1973). Electrical signs of selective attention in the human brain. Science, 182, 177-180.

Freedman, R., Waldo, M., Waldo, C.I., \& Wilson, J.R. (1987). Genetic influences on the effects of alcohol on auditory evoked potentials. Alcohol, 4, 249-253.

Guterman, Y., \& Josiassen, R.C. (1994). Sensory gating deviance in schizophrenia in the context of task related effects. International Journal of Psychophysiology, 18, 1-12.

Guterman, Y., Josiassen, R.C., \& Bashore, T.R.J. (1992). Attentional influence on the P50 component of the auditory eventrelated brain potential. International Journal of Psychophysiology, 12, 197-209.

Jasper, H.H. (1958). The ten-twenty electrode system of the International Federation. Electroencephalography and Clinical Neurophysiology, 10, 371-375.

Jerger, K., Biggins, C., \& Fein, G. (1992). P50 suppression is not affected by attentional manipulations. Biological Psychiatry, 31, 365-377.

Judd, L., McAdams, L., Budnick, B., \& Braff, D. (1992). Sensory gating deficits in schizophrenia: New results. American Journal of Psychiatry, 149, 488-493.

Kisley, M.A., Olincy, A., \& Freedman, R. (2001). The effect of state on sensory gating: Comparison of waking, REM and nonREM sleep. Clinical Neurophysiology, 112, 1154-1165.

Light, G.A., \& Braff, D.L. (1998). The "incredible shrinking” P50 event-related potential. Biological Psychiatry, 3, 918-920.

Mangun, G.R., \& Hillyard, S.A. (1995). Mechanisms and models of selective attention. In M.G.H. Coles \& M.D. Rugg (Eds.), Electrophysiology of mind. Event-related potentials and cognition. Oxford: Oxford University Press.

Näätänen, R., \& Teder, W. (1991). Attention effects on the auditory event-related potential. Acta Otolaryngol Suppl, 491, 161-166.

Nagamoto, H.T., Adler, L.E., Waldo, M.C., \& Freedman, R., (1989). Sensory gating in schizophrenics and normal controls: Effects of changing stimulation interval. Biological Psychiatry, 25, 549-561.

Neylan, T.C., Fletcher D.J., Lenoci, M., McCallin, K., Weiss, D.S., Schoenfeld, F.B., Marmar, C.R., \& Fein, G. (1999). Sensory gating in chronic posttraumatic stress disorder: Reduced auditory P50 suppression in combat veterans. Biological Psychiatry, 46, 1656-64

Picton, T.W., Bentin, S., Berg, P., Donchin, E., Hillyard, S.A., Johnson jr., R., Miller, G.A., Ritter, W., Ruchkin, D.S., Rugg, M.D., \& Taylor, M.J. (2000). Guidelines for using human event-related potentials to study cognition: Recording standards and publication criteria. (Committee Report). Psychophysiology, 37, 127-152.

Smith, D.A., Boutros, N.N., \& Schwarzkopf, S.B. (1994). Reli- 
ability of P50 auditory event related potential indices of sensory gating. Psychophysiology, 31, 495-502.

Waldo, M.C., \& Freedman, R. (1986). Gating of auditory evoked responses in normal college students. Psychiatry Research, 19, 233-239.

van Luijtelaar, E.L., Miller, C.A., Coenen, A.M., Drinkenburg, W.H., \& Ellenbroek, B.A. (1998). Differential effects of nonREM and REM sleep on sensory gating in rats. Acta Neurobiol Exp (Warsz), 58, 263-70

White, P.M., \& Yee, C.M. (1997). Effects of attentional and stressor manipulations on the P50 gating response. Psychophysiology, 34, 703-711.

Yee, C.M., \& White, P.M. (2001). Experimental modification of P50 suppression. Psychophysiology, 38, 531-539.
Gilles van Luijtelaar

NICI-Biological Psychology

University of Nijmegen

PO Box 9104

6500 HE Nijmegen

The Netherlands

Tel. +31 24 361-5621

Fax +31 24 361-6066

E-mail luijtelaar@nici.kun.nl 